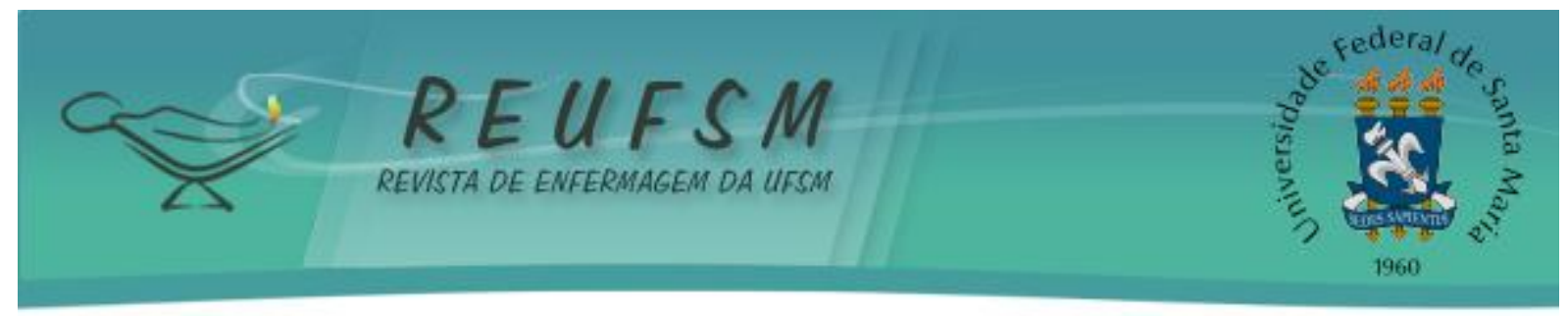

ARTIGO ORIGINAL

\title{
ATIVIDADES GERENCIAIS DO ENFERMEIRO NA ESTRATÉGIA DE SAÚDE DA FAMÍLIA
}

NURSE'S MANAGERIAL ACTIVITIES IN FAMILY HEALTH STRATEGIES

ACTIVIDADES DE GERENCIA DE LO ENFERMERO EM LA ESTRATEGIA DE

SALUD DE LA FAMILIA

\author{
Davi Antonio Brondani Junior ${ }^{1}$ \\ Rita Maria Heck ${ }^{2}$ \\ Teila Ceolin ${ }^{3}$ \\ Carmem Rosane da Silva Viegas ${ }^{4}$
}

RESUMO: o objetivo deste artigo foi identificar as atividades realizadas pelo enfermeiro atuante na Estratégia de Saúde da Família, enfatizando as ações gerenciais. Este trabalho consistiu em um estudo qualitativo. Os sujeitos do estudo foram cinco enfermeiros que atuavam na ESF em municípios localizados na região sul do Rio Grande do Sul. Os dados foram coletados no período de outubro a novembro de 2008 , através da técnica de autorrelato estruturado e foram analisados por temáticas. As ações em saúde realizadas pelos enfermeiros estão de acordo com o preconizado pelo Ministério da Saúde, com exceção das atividades de vigilância em saúde. A função de enfermeiro ainda possui contradições, pois alguns compreendem as atividades gerenciais como desvio do cuidado ao usuário, considerando a assistência e gerenciamento, funções dicotômicas, demonstrando o despreparo em realizar esta tarefa.

Descritores: Enfermagem; Saúde da família; Gerência; Atenção primária à saúde.

ABSTRACT: the purpose of this paper was to identify the nurse's Family Health Strategy (FHS) activities. It is a qualitative study, and the subjects were 5 nurses from different counties in southern Rio Grande do Sul working in FHS. Data were collected between October and November 2008, using the technique of structured self-report and were analyzed for themes. Their activities are in agreement with Health Ministry recommendations, except for health surveillance activities. The nurse role is still contradictory, since some still think of managerial activities as being a deviation in users' care, considering assistance and management as two-sided roles, thus showing a lack of preparation in dealing with this task.

Descriptors: Nursing; Family health; Management; Primary health care.

RESUMEN: el objetivo de este artículo fue identificar las actividades realizadas por los enfermeros que trabajan en la Estrategia de Salud de la Familia (ESF), enfatizando las acciones gerenciales. Este trabajo consistió en un estudio cualitativo. Los sujetos fueron cinco enfermeros que trabajan en el ESF en las localidades ubicadas en la región Sur del Rio Grande do Sul. Los datos fueron recolectados entre octubre y noviembre de 2008, utilizando la técnica de la estructura de autoinforme y se los analizaron por temas. Las acciones en salud realizadas por los enfermeros están de acuerdo con las recomendaciones del Ministerio de Salud, excepto las actividades de vigilancia en salud. El papel del

\footnotetext{
${ }^{1}$ Secretaria Municipal de Saúde/Três Coroas/RS. E-mail: davibrondani@hotmail.com.

2 Doutora em Enfermagem. Professora Associada da Faculdade de Enfermagem (FEn) da Universidade Federal de Pelotas (UFPel). Orientadora da pesquisa. E-mail: heck@ufpel.edu.br.

${ }^{3}$ Mestre em Enfermagem. Professora Assistente da FEn/UFpel. E-mail: teila.ceolin@ig.com.br.

4 Especialista em Estratégia da Família. Coordenadora da Atenção Básica. Capão do Leão/RS. E-mail: crsviegas@ig.com.br
} 


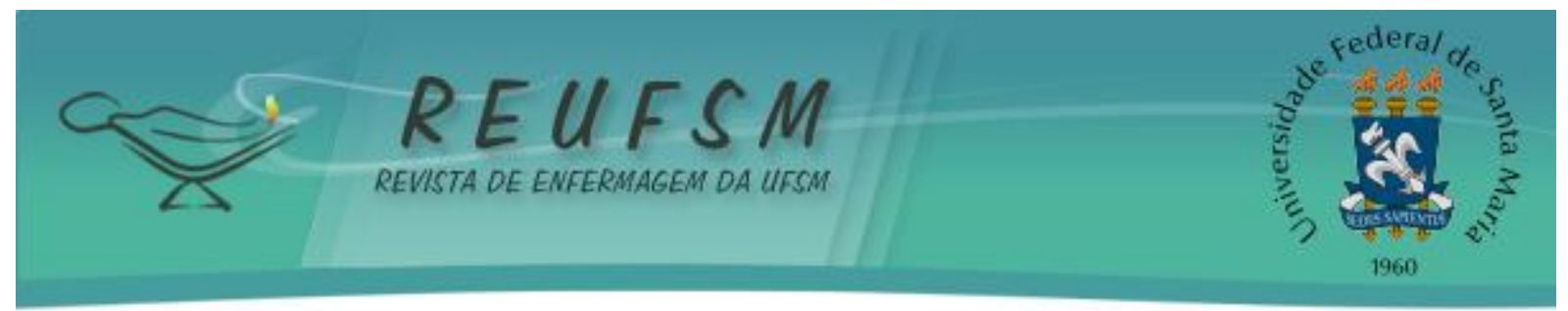

enfermero aún tiene contradicciones, pues algunos comprenden las actividades gerenciales como un alejamiento del cuidado al usuario, considerando la asistencia y la gerencia funciones dicotómicas, lo que demuestra la falta de preparación para realizar esta tarea.

Descriptores: Enfermería; Salud de la familia; Gerencia; Atención primaria de salud.

\section{INTRODUÇÃO}

Anteriormente à Constituição Federal de 1998, o acesso à saúde oferecido pelo Estado ocorria através da Previdência Social apenas para os trabalhadores da economia formal e seus dependentes, não havendo, portanto, caráter universal na atuação. ${ }^{1} \mathrm{~A}$ desarticulação dos serviços de saúde, o acesso somente aos previdenciários e os evidentes prejuízos à saúde da população decorrentes do modelo vigente, geraram na comunidade de profissionais da saúde, de sanitaristas, sindicatos e da própria sociedade brasileira, um movimento na direção de uma reforma sanitária e de uma transformação dos paradigmas do sistema de saúde. ${ }^{2}$

O Movimento da Reforma Sanitária que iniciou na década de 70 buscou a modificação do enfoque assistencial procurando ampliar a rede de serviços na atenção primária à saúde. Em março de 1986, ocorreu a VIII Conferência Nacional de Saúde, um marco para as políticas de saúde do país, na qual foram lançadas as bases doutrinárias do Sistema Único de Saúde (SUS), com acesso integral e universal a saúde para todos os brasileiros. $^{2}$

Desde a criação do SUS, pela constituição de 1988, ocorreram várias alterações nas políticas de saúde, em busca de um cuidado integral e humanizado. Em 1994 houve a criação do Programa Saúde da Família, pelo Ministério da Saúde. A implantação do programa resultou na melhora dos indicadores de saúde na população acompanhada, o qual passou a ser uma estratégia de reorganização do modelo de assistência à saúde, denominada Estratégia Saúde da Família (ESF), tendo como um de seus objetivos, o desenvolvimento de vínculos com as famílias do território adscrito para realização do cuidado, visando à reorganização da Atenção Básica no País de acordo com os preceitos do SUS..$^{3-4}$

A ESF possui, na sua composição mínima, um enfermeiro, um médico, um auxiliar ou técnico de enfermagem e até doze agentes comunitários de saúde (ACS). ${ }^{4}$ Essa equipe é responsável pela promoção, prevenção da saúde dos usuários assistidos, não se descuidando do aspecto curativo, reabilitador e com alta resolubilidade. Para que estas ações ocorram é necessário o desenvolvimento de atividades interdisciplinares e intersetoriais.

Entre as competências do enfermeiro da Unidade de Saúde da Família (USF), estão as atividades de planejamento, gerenciamento, coordenação, execução e avaliação. ${ }^{5}$ As ações gerenciais devem ser integradas com o trabalho da equipe, resultando em transformações em prol da qualidade do cuidado integral e da promoção da saúde da população assistida. ${ }^{6}$

O enfermeiro deve estar sempre em busca de uma assistência qualificada e de um ambiente de trabalho adequado. ${ }^{7}$ A educação em saúde é uma das suas funções, a qual pode ser desenvolvida durante a consulta de enfermagem, na sala de espera, com grupos terapêuticos, por meio de visitas domiciliares, reuniões comunitárias e atividades desenvolvidas nas escolas, entre outros. ${ }^{8}$

O trabalho na ESF exige principalmente do enfermeiro, uma liderança capaz de articular estratégias institucionais com enfoque na promoção da saúde e efetivação dos princípios do SUS. ${ }^{9}$ O profissional deve ter atitudes e habilidades em prol do coletivo, 


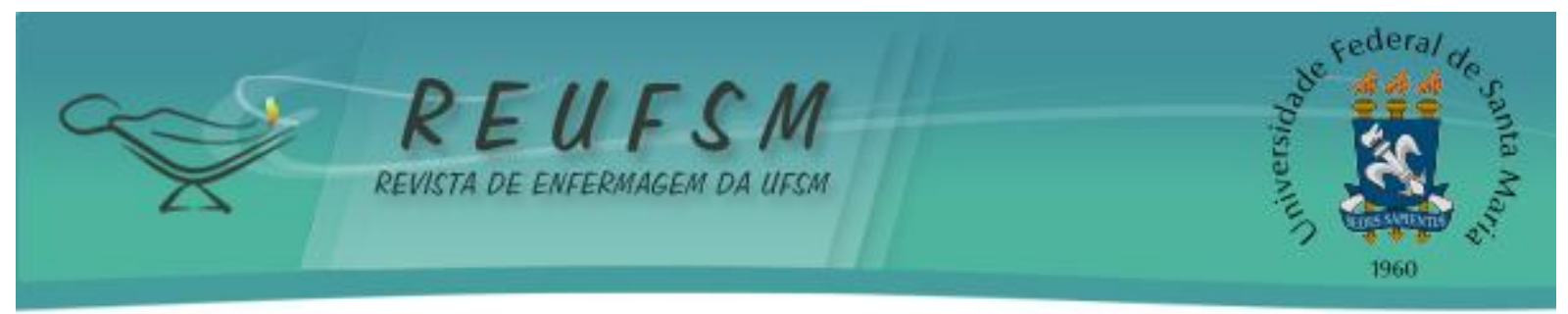

gerenciando e organizando a USF na qual atua, conciliando recursos humanos e materiais para realização da assistência. ${ }^{10}$

Diante deste contexto, este estudo teve como objetivo identificar as atividades realizadas pelo enfermeiro atuante na Estratégia de Saúde da Família, enfatizando as ações gerenciais.

\section{MÉTODO}

Esta pesquisa consistiu em um estudo qualitativo, do tipo exploratório ${ }^{11-12}$ realizado em uma Universidade Pública da região sul do Rio Grande do Sul.

Os sujeitos foram cinco enfermeiros que atuavam na Estratégia de Saúde da Família (ESF) em municípios localizados na região sul do Rio Grande do Sul e que cursavam a Especialização Multiprofissional em Saúde da Família oferecida pela Universidade Pública. A escolha seguiu os seguintes critérios: enfermeiros que se dispusessem e manifestassem interesse em participar do estudo; que atuassem na ESF; estivessem frequentando o curso de especialização e autorizassem a divulgação e publicação dos dados.

O total de enfermeiros que cursavam a Especialização Multiprofissional em Saúde da Família era 46. Destes foram selecionados 14 que atuavam na ESF, em diferentes municípios. No dia agendado para entrega dos questionários, somente 6 enfermeiros compareceram nas atividades da especialização. Entre os respondentes um não devolveu o instrumento de pesquisa fornecido, totalizando uma amostra final de cinco entrevistados, os quais atuavam em quatro municípios da região sul do Rio Grande do Sul.

Os dados foram coletados no período de outubro a novembro de 2008 , por meio da técnica de autorrelato estruturado ${ }^{13}$, o qual foi entregue aos enfermeiros em uma data agendada previamente. 0 questionário constava de dados do perfil e de cinco questões abertas. As questões foram: quais as atividades desenvolvidas na instituição de trabalho?; quais são as funções do enfermeiro?; você se considera enfermeiro gerente da sua instituição de trabalho?; descreva as atividades gerenciais realizadas na sua última semana de trabalho detalhando os dias da semana e turnos, especificando o tempo dedicado a cada uma delas; quais são as possíveis dificuldades encontradas ao gerenciar a USF?

Após a coleta, os dados foram agrupados de forma comparativa de acordo com cada questão abordada. Após foram analisados, gerando dois temas: atividades e funções do enfermeiro; atividades gerenciais e possíveis dificuldades encontradas pelos enfermeiros na sua execução. ${ }^{11}$ Os sujeitos foram identificados pela inicial "E", seguido de números cardinais. Ex: E1, E2.

O projeto foi aprovado pelo Comitê de Ética em Pesquisa da Universidade Católica de Pelotas, parecer $n^{\circ} 57 / 2008$. Foram atendidos os princípios éticos da Resolução $196 / 96,{ }^{14}$ sendo que todos os respondentes assinaram o Termo de Consentimento Livre e Esclarecido.

\section{RESULTADOS E DISCUSSÃO}

Em relação ao tempo de vínculo institucional com a Unidade de Saúde, este variou de três meses a 10 anos, sendo que a maioria possuía menos de um ano. Quanto ao tempo de atuação do enfermeiro na ESF, este variou entre três meses a seis anos, com predominância de trabalhadores com menos de um ano de atuação. A idade dos sujeitos variou entre 24 e 42 anos. 


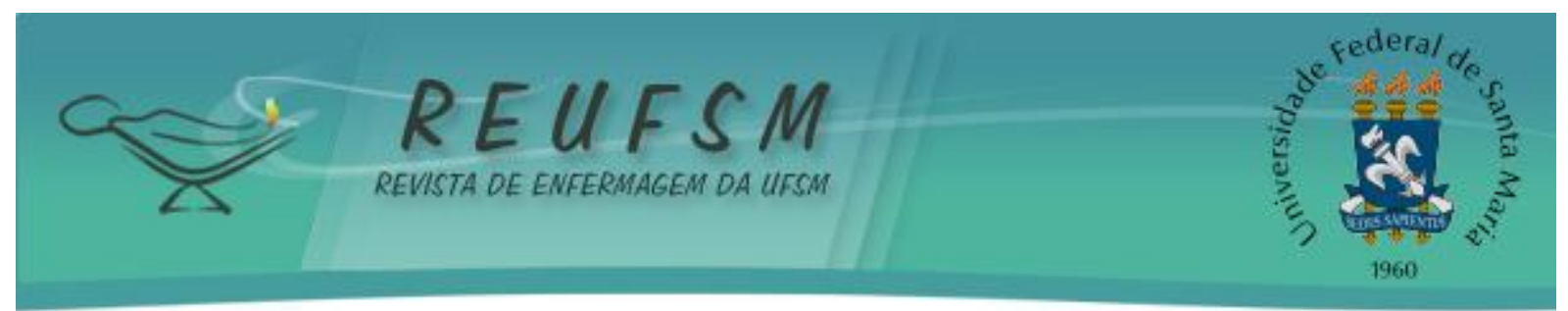

A predominância do sexo feminino encontrada nesta pesquisa reforça a prevalência de mulheres da profissão, pois somente um dos sujeitos era do sexo masculino, dado semelhante encontrado em outra pesquisa. ${ }^{15}$

\section{Atividades e funções do enfermeiro}

O Ministério da Saúde preconiza como funções específicas do enfermeiro na ESF a realização da assistência integral em todas as fases do desenvolvimento humano conforme protocolos ou outras normativas técnicas estabelecidas; consulta de enfermagem; solicitação de exames complementares; prescrição de medicações; planejamento, gerenciamento, coordenação e avaliação das ações desenvolvidas pelos ACS; participação do gerenciamento dos insumos necessários para o adequado funcionamento da USF, entre outras funções. ${ }^{4}$

0 trabalho do enfermeiro na ESF se constitui no monitoramento das condições de saúde, como núcleo da atenção de enfermagem, seja no atendimento individual ou no atendimento grupal; no levantamento e monitoramento de problemas de saúde (no enfoque de risco ou de vulnerabilidade) sendo que estes deverão estar articulados à intervenção nos agravos de ordem patológica (pautados no saber da clínica) e no exercício de uma prática de enfermagem comunicativa, no sentido dialógico e emancipatório, buscando a ampliação da autonomia dos sujeitos. ${ }^{16}$ Assim, discorrer sobre as práticas de enfermagem implica no entendimento de que a enfermagem, enquanto prática social, busca responder às exigências sociais e de saúde de uma determinada época e espaço social. ${ }^{17}$

Ao abordar as atividades desenvolvidas no local de trabalho, a maioria dos enfermeiros refere realizar tanto as assistenciais, quanto a gerencial. Em relação as atividades assistenciais, os sujeitos relataram as seguintes: pré-natal de baixo risco, coleta de citopatológico para prevenção do câncer de colo uterino, revisão puerperal, puericultura, imunizações, coleta do teste do pezinho, notificações SIM, SINASC, SINAN; consulta de enfermagem, aferição da pressão arterial sistêmica e glicemia, curativos, administração de medicamentos, realização de curativo, visitas domiciliares, atendimento domiciliar a pacientes debilitados, coordenação dos grupos de hipertensos e diabéticos, grupos fora da USF junto à comunidade nos projetos existentes no bairro, realização de campanhas, palestras em escolas, orientações na pré-sala (recepção) junto aos usuários. Quanto às atividades gerenciais, estas são apresentadas e discutidas no subtítulo: Atividades gerenciais e dificuldades encontradas pelos enfermeiros na sua execução.

Um estudo realizado com enfermeiros da Secretaria Municipal de Saúde de Goiânia, também evidenciou que dentre o conjunto de atividades do enfermeiro, a maioria são de assistência à saúde nos programas do Ministério da Saúde. ${ }^{18}$

$O$ trabalho do enfermeiro na ESF, segundo os autorrelatos, ocorre basicamente em dois ambientes: a USF e a comunidade. Em ambos apóia e supervisiona o trabalho do agente comunitário de saúde (ACS) e do auxiliar de enfermagem, atendendo aos usuários que buscam a USF e os que necessitam de cuidados domiciliares ${ }^{19}$, considerando o contexto local.

As atividades do enfermeiro devem estar voltadas para a população assistida. Deve-se atuar em equipe junto com a comunidade, considerando o contexto e as necessidades locais, para que as soluções sejam as mais adequadas. Apenas dois enfermeiros mencionaram realizar ações fora da USF, evidenciando a persistência da influência do modelo biomédico, focado na doença e a necessidade emergente da realização de ações visando a promoção da saúde nos espaços da comunidade. 


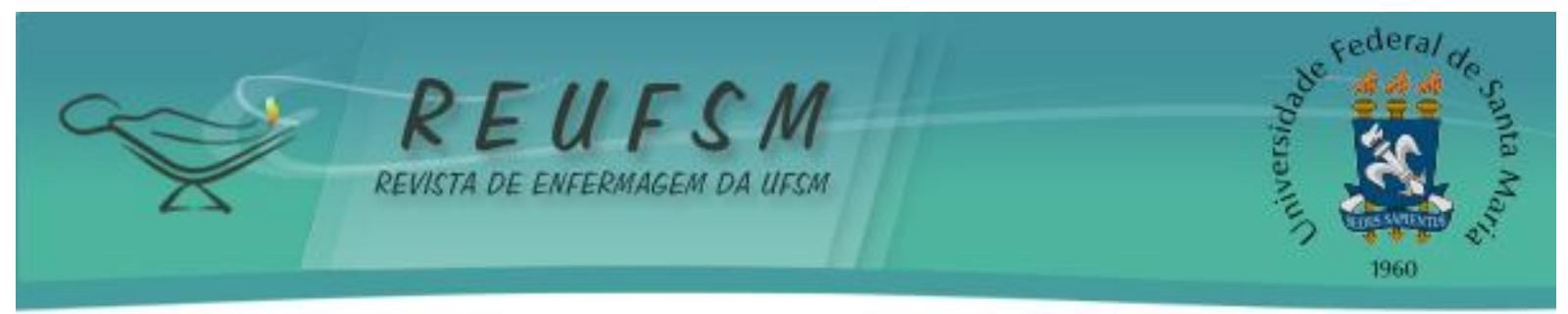
seguir:

A realização de atividades intersetoriais pode ser evidenciada por meio do relato a

[...] contato com escola do bairro e Conselho Tutelar, sobre casos de drogas com adolescentes do bairro. (E1)

A prática do trabalho interdisciplinar e intersetorial é importante para o desenvolvimento das ações da equipe de saúde da família, pois possibilita a execução do cuidado integral e resolutivo, sendo também uma forma de solucionar os problemas da população de forma integrada, considerando a sua totalidade. ${ }^{20}$

As atividades mencionadas pelos enfermeiros estão de acordo com a Portaria 648 e os programas do Ministério da Saúde. ${ }^{4}$ As ações destinadas à saúde da mulher e da criança foram citadas por todos os enfermeiros, já a vigilância em saúde, foi mencionada por apenas um enfermeiro. Estes dados são semelhantes ao encontrado em outra pesquisa ${ }^{21}$, a qual evidenciou a predominância destas ações e pouca atuação na vigilância em saúde.

As reuniões de equipe são atividades elencadas por todos os enfermeiros, fazendo parte da rotina de trabalho, entretanto não amenizam as dificuldades relacionadas ao funcionamento da equipe, como podemos observar nos seguintes relatos.

[...] há divergências com alguns profissionais da equipe, em razão do perfil dos profissionais da ESF. Falta de iniciativa, empenho, comprometimento de alguns profissionais com a ESF. (E3)

A falta de entendimento com membros da equipe que não tem entendimento suficiente quanto ao funcionamento da estratégia de saúde da família. (E5)

Geralmente, os conflitos que surgem durante o trabalho de enfermagem em uma unidade de saúde são devido à falha na comunicação, diferenças de valores e de personalidades. Por conseguinte, o enfermeiro deve saber gerenciar esses conflitos para que o trabalho da equipe possa resultar na assistência de qualidade à população.

As visitas domiciliares foram mencionadas por todos os enfermeiros deste estudo, o que poderia ser sinônimo de prevenção, proteção e promoção da saúde. Porém, nenhum dos respondentes citou sobre as ações feitas durante as visitas, não sendo possível inferir sobre a qualidade dessa atividade.

0 trabalho realizado com grupos também foi citado por todos os sujeitos, mas somente dois comentaram sobre o que era desenvolvido nestes grupos.

[...] atividades de grupo visando a melhoria na qualidade de vida das gestantes, hipertensos, diabéticos, idosos, adolescentes, através de palestras dinâmicas. Realizar atendimento visando à promoção e prevenção da saúde das pessoas nas diferentes faixas etárias durante os atendimentos da demanda (na puericultura, précâncer, no pré-natal,...), inclusive nas residências durante as visitas. (E4)

[...] coordenação dos grupos de hipertensos e diabéticos. (E2)

Dentre as estratégias de educação em saúde, destacam-se as dinâmicas de grupo. ${ }^{22} 0$ grupo é evidenciado como possibilidade de multiplicação dos recursos assistenciais e construção de novos e eficazes modelos que possam ser mais abrangentes, 


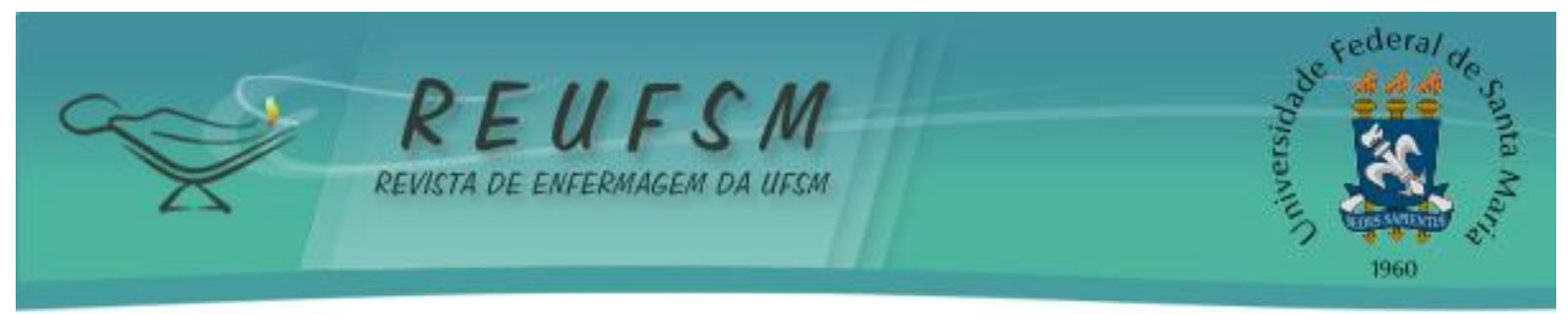

multidimensionados e acessíveis. ${ }^{23}$ A comunicação intermediada através das atividades de educação em saúde pode ser vista como um mecanismo de troca entre o saber científico e o popular, proporcionando a socialização do saber científico e o reconhecimento do saber popular. ${ }^{22}$

As ações de educação em saúde foram referidas somente por um dos enfermeiros, ressaltando as dificuldades em realizá-la, como destacamos no relato a seguir:

[...] demanda reprimida, ficando prejudicada a prevenção $e$ educação em saúde da ESF, [...] comunidade local com dificuldade de assimilar e aceitar a ESF [...] (E1).

A educação em saúde é uma ação extremamente importante no que diz respeito à Estratégia Saúde da Família, devendo levar em consideração o saber do usuário, o contexto no qual está inserido e a sua cultura.

A execução de atividades de educação permanente para os ACS e para a equipe de enfermagem foi citada somente por um dos enfermeiros. O Ministério da Saúde afirma que estas atividades são de responsabilidade específica do enfermeiro. ${ }^{4}$

Um estudo realizado com enfermeiros da Secretaria Municipal de Saúde de Goiânia encontrou que $29,5 \%$ das atividades desenvolvidas eram ações de educação para a comunidade e $27,3 \%$ ações de educação em serviço e capacitação para a equipe de saúde. $^{18}$

Além das atividades que são de competência dos enfermeiros, um sujeito destacou a execução de funções que não lhe competem.

[...] faço procedimentos de técnico de enfermagem e de enfermeiro, além de outros como: burocrata, serviço social, secretária, na falta de faxineira se faz até faxina. (E5)

A partir deste relato devemos realizar o questionamento: na falta de outros trabalhadores, o enfermeiro deve realizar todos os outros serviços?

Ao serem questionados sobre o que consideram suas funções, além das mencionadas anteriormente, também destacaram as atividades gerenciais.

Todas de sua competência, incluindo gerência da USF, responsabilidade técnica perante o COREN e coordenação dos ACS. Atuar na promoção, prevenção e educação junto aos setores locais, criando parcerias no bairro. (E1)

0 enfermeiro deve realizar atividades profissionais voltadas para o contexto da vida do usuário. É importante salientar que as funções do enfermeiro devem priorizar o cuidado terapêutico ao cliente, respaldadas pela Lei do Exercício Profissional, sejam elas gerenciais ou assistenciais.

\section{Atividades gerenciais e dificuldades encontradas pelos enfermeiros na sua execução}

0 enfermeiro tem como competências realizar funções que priorizem os aspectos individuais e coletivos dos usuários do SUS, principalmente em relação à promoção, à proteção e à prevenção da saúde. Os planos de ação devem ser realizados conjuntamente entre profissional e usuário/comunidade, considerando seus saberes e práticas de cuidado à saúde, o contexto do território e o diagnóstico de saúde local. 


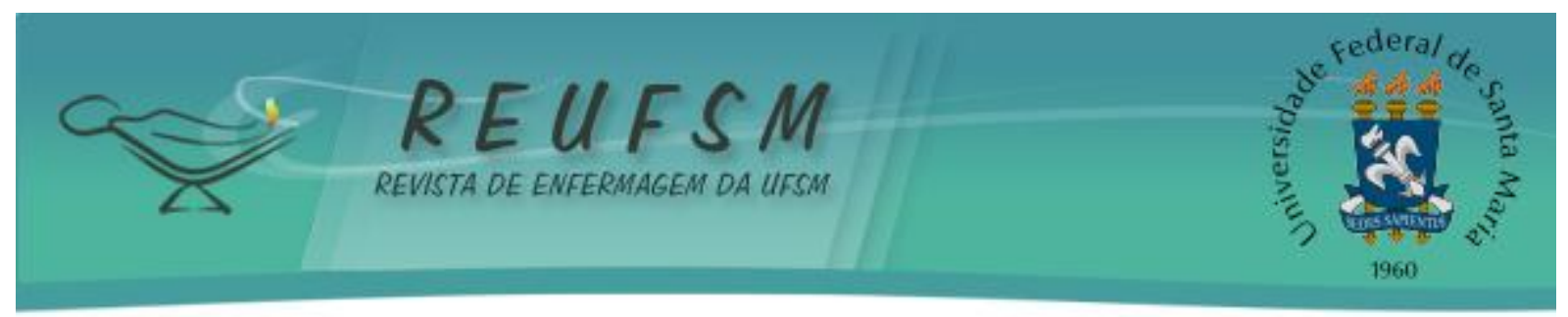

Os enfermeiros descreveram as seguintes ações gerenciais: coordenação de equipe, reuniões com equipe, realizar educação continuada para profissionais técnicos e ACS, inclusive promover estas atividades para si e para os médicos, através da busca de capacitações, preenchimento de relatórios, gerenciamento da USF, planejamento de atividades, organização e administração de materiais, coordenação/gerência da USF, supervisão dos ACS, responsabilidade técnica perante o COREN.

Quanto a realização do planejamento das ações, somente um enfermeiro referiu realiza-lo. As funções gerenciais fazem parte da rotina do enfermeiro, assim como as funções assistenciais, uma vez que ambas estão interligadas. Para que ocorra um bom gerenciamento é necessário planejamento, ou seja, deve-se estabelecer um plano de atuação envolvendo todos os profissionais da equipe ${ }^{24}$ e a comunidade.

As atividades gerenciais do enfermeiro devem estar voltadas à assistência da população, a qual necessita ser o principal objetivo na organização do gerenciamento, uma vez que todo o processo de trabalho deve ser centrado no cuidado individual e coletivo.

Em relação ao tempo destinado às atividades gerenciais realizadas pelos enfermeiros deste estudo percebe-se que, na organização do trabalho, existem diferenças entre os enfermeiros. Um enfermeiro não especificou funções gerenciais durante uma semana de trabalho, registrando somente atividades assistenciais. Os demais, que responderam realizar funções gerenciais, especificaram o total de tempo utilizado para fazê-las, variando entre $7 \mathrm{~h} 35^{\prime}$ e $14 \mathrm{~h}$ e $30^{\prime}$ durante uma semana de trabalho, considerando a carga de trabalho semanal de $40 \mathrm{~h}$.

Nos relatos dos enfermeiros a seguir, percebe-se que todos realizam ações gerenciais embora, em alguns casos, isto não seja aceito ou não ocorra da maneira preconizada.

Sim, mas não gostaria, pois em certas situações o enfermeiro passa assumir responsabilidades e atribuições que não são dele, ficando dessa forma o trabalho do enfermeiro prejudicado, havendo um distanciamento entre o profissional e a pessoa a ser cuidada. (E3) Sim, mas só para gerenciar os problemas da USF. Não há autonomia para tomar decisões, para mudar a forma de trabalho na USF $e$ equipe de ESF. (E2)

A gerência deve ser um instrumento do processo de trabalho nos serviços de saúde, pois ela implica na tomada de decisões que visam ações que possibilitam intervenções no trabalho em saúde. ${ }^{25}$

Um dos enfermeiros citou que não recebeu preparo adequado durante sua formação acadêmica para a realização de ações gerenciais, como descrito a seguir:

sim, esta é uma atividade diária a ser realizada pelo enfermeiro, independente da sua vontade. A instituição exige. Deixo aqui meu comentário de que a faculdade não nos prepara suficientemente para realizar estas atividades. (E4)

O enfermeiro encontra em seu trabalho nas unidades de ESF grandes dificuldades. A maior delas é a falta de capacitação para a realização das tarefas. As competências e habilidades específicas para o desenvolvimento das ações gerenciais devem ser desenvolvidas durante o processo de formação, sendo permanentemente construídas no cotidiano, podendo colaborar na capacitação do profissional. ${ }^{18}$ 


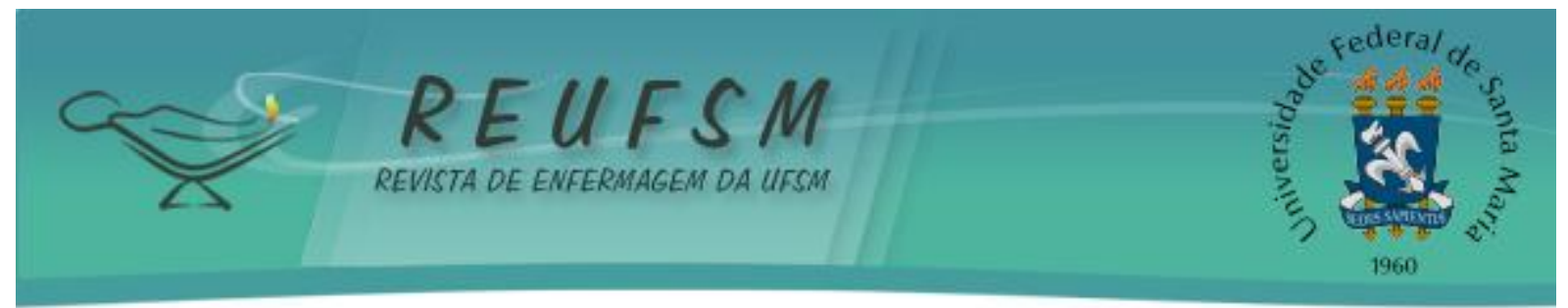

A seguir, podemos observar nos relatos dos sujeitos da pesquisa as dificuldades encontradas para realização das ações gerenciais.

Realizar atividades que não são suas atribuições, falta de estrutura física da USF para promover atendimento de qualidade. Há divergências com alguns profissionais da equipe, em razão do perfil dos profissionais da ESF. Falta de iniciativa, empenho, comprometimento de alguns profissionais com a ESF. (E3)

[...] não há autonomia para tomar decisões, para mudar a forma de trabalho na USF e equipe de ESF. (E2)

[...] em alguns casos falta de apoio de gestores. O fator político, na maioria das vezes interfere negativamente no trabalho da USF. (E2)

Sabe-se que as atividades gerenciais e assistenciais estão interligadas, caso contrário o serviço de saúde fica prejudicado. Percebemos que a resolubilidade das ações na ESF depende da capacidade do enfermeiro em planejá-las. Para que os problemas sejam resolvidos de maneira positiva, equipe e comunidade devem apresentar soluções possíveis de acordo com a realidade da comunidade ou do indivíduo. ${ }^{26}$

0 enfermeiro necessita de um perfil baseado nas atitudes, para transformar, inovar, transcender e tornar as organizações de saúde mais resolutivas. ${ }^{15}$ É importante ressaltar que a organização faz parte de qualquer processo de trabalho, seja gerencial ou assistencial. Foi possível observar através dos relatos dos enfermeiros, que há grandes dificuldades no que diz respeito ao gerenciamento de uma unidade de saúde.

As principais dificuldades nas atividades gerenciais do enfermeiro referidas em um estudo 9 foram: conciliar às atividades assistenciais as gerenciais, a estrutura física, a falta de capacitação, a "politicagem", a pouca autonomia, a falta de compromisso por parte de alguns profissionais e a ausência de apoio da Secretaria da Saúde.

As atividades citadas pelos sujeitos E2 e E3, não condizem com a real função de um enfermeiro, o qual deve possuir autonomia para planejar e executar seu trabalho, envolvendo a equipe e buscando a qualidade da assistência prestada. A realização de outras atividades que não lhe competem, assim como a falta de apoio do gestor, pode prejudicar a qualidade do cuidado prestado ao usuário. Além disso, os enfermeiros devem ser preparados em relação ao gerenciamento em saúde pública.

Neste contexto, uma das importantes interseções entre saúde e educação, é a adequação do ensino, conhecimentos produzidos e serviços prestados à população com base nas necessidades sociais, preconizando formar um enfermeiro generalista, humanista, crítico e reflexivo. ${ }^{18}$

\section{CONSIDERAÇÕES FINAIS}

A função de enfermeiro ainda possui contradições, pois alguns compreendem as atividades gerenciais como desvio do cuidado ao usuário considerando, muitas vezes, a assistência e as ações gerenciais como funções dicotômicas.

$O$ enfermeiro atuante na ESF, para realização de um cuidado integral e humanizado, deve estar articulado com a equipe, levando em consideração o contexto sócio-econômico-cultural no qual o indivíduo e sua família estão inseridos, visando a promoção da saúde e melhoria da qualidade de vida.

Para isso, as ações gerenciais devem fazer parte da prática deste profissional, necessitando ser desenvolvida durante a formação acadêmica para que este possa qualificar a assistência prestada à população. 


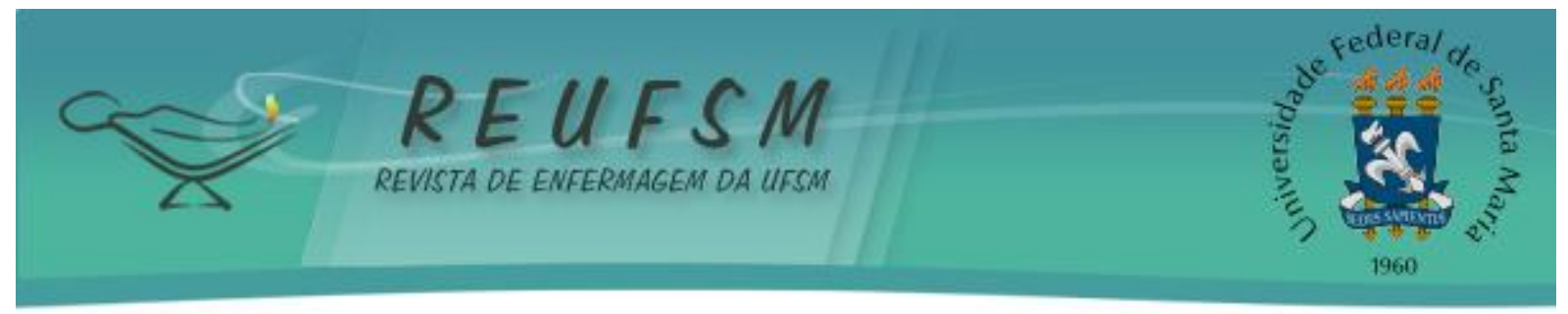

\section{REFERÊNCIAS}

1. Conselho Nacional de Secretários de Saúde (BR). Sistema Único de Saúde. Brasília: CONASS; 2007.

2. Conselho Nacional de Secretários de Saúde (BR). SUS 20 anos. Brasília: CONASS; 2009.

3. Ministério da Saúde (BR). Guia Prático do Programa Saúde da Família. Brasília: Ministério da Saúde; 2001.

4. Ministério da Saúde (BR). Departamento de Atenção Básica. Portaria $n^{\circ} 648$, de 28 de março de 2006. Brasília: Ministério da Saúde; 2006.

5. Ministério da Saúde (BR). Secretaria de Gestão Estratégica e Participativa. A construção do SUS: histórias da Reforma Sanitária e do Processo Participativo. Brasília: Ministério da Saúde; 2006.

6. Galvão CM, Trevizan MA, Sawada NO. A Liderança do Enfermeiro no Século XXI: algumas considerações. Rev Esc Enferm USP. 1998; 32(4):302-6.

7. Lunardi Filho WD. 0 mito da subalternidade do trabalho de enfermagem à medicina. Pelotas: Ed. Universitária/UFPel, Florianópolis: UFSC; 2000.

8. Nascimento MS, Nascimento MAA. Prática da enfermeira no Programa de Saúde da Família: a interface da vigilância da saúde versus as ações programáticas em saúde. Ciênc saúde coletiva. 2005;10(2):333-45.

9. Ximenes Neto FRG, Sampaio JJC. Processo de ascensão ao cargo e as facilidades e dificuldades no gerenciamento do território na Estratégia Saúde da Família. Rev bras enferm. 2008; 61(1): 36-45.

10. Baldassare RM, Ciampone MHT. A construção de competências para o gerenciamento em enfermagem: a percepção dos alunos dos sétimo e oitavo semestres de graduação em enfermagem. Rev adm saúde. 2007; 9(35):47-54.

11. Minayo MCS. O desafio do conhecimento. $11^{\text {a }}$ ed. São Paulo/Rio de Janeiro: HucitecAbrasco; 2008.

12. Triviños ANS. Introdução à pesquisa em ciências sociais: a pesquisa qualitativa em educação. São Paulo: Atlas; 2008.

13. Polit DF, Beck CT, Hungler BP. Fundamentos de pesquisa em enfermagem: métodos, avaliação e utilização. $5^{\text {a }}$ ed. Porto Alegre: Artmed; 2004.

14. Ministério da Saúde (BR). Conselho Nacional de Saúde. Decreto $n^{\circ} 93.9333$ de 14 de janeiro de 1987, Resolução 196/96. Diretrizes e normas reguladoras de pesquisas envolvendo seres humanos. Brasília; 1996.

15. Lazzarotto EM, Cardoso GM, Nazzari RK, Bozza MS. Atitudes requeridas para gerenciar as unidades básicas de saúde. Arq Ciênc Saúde Unipar. 2003; 7(2):135-44.

16. Peduzzi M. A inserção do enfermeiro na equipe de saúde da família na perspectiva de promoção de saúde. In: Anais do $1^{\circ}$ Seminário Estadual: o enfermeiro no programa de saúde da família; 2000 nov. 9-11; São Paulo. São Paulo: Secretaria de Estado da Saúde; 2000. p.01-11. 


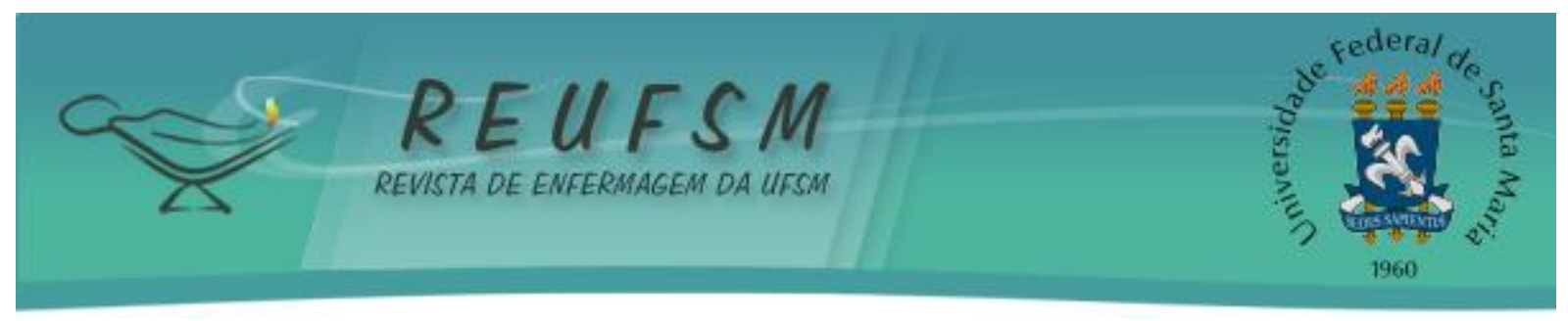

17. Ermel RC, Fracolli LA. O trabalho das enfermeiras no Programa de Saúde da Família em Marília/SP. Rev Esc Enferm USP. 2006; 40(4):533-9.

18. Weirich CF, Munari DB, Mishima SM, Bezerra ALQ. O trabalho gerencial do enfermeiro na Rede Básica de Saúde. Texto \& contexto enferm. 2009; 18(2):249-57.

19. Santos MR. Atribuições Legais do Enfermeiro no Programa de Saúde da Família: Dificuldade e Facilidades. Boletim da Saúde (Porto Alegre). 2003; 17(2):37-40.

20. Junqueira LP. Novas Formas de Gestão na Saúde: descentralização e intersetorialidade. Saúde Soc. 1997; 6(2):31-46.

21. Ceolin T, Heck RM, Casarin ST, Ceolin AR. Processo de trabalho dos enfermeiros na Estratégia de Saúde da Família. Enfermería Comunitária. 2009; 5(1):[aproximadamente 12 p.].

22. Maffacciolli R, Lopes MJM. Educação em saúde: a orientação alimentar através de atividades de grupo. Acta paul enferm. 2005;18(4):439-45

23. Zimerman DE, Osório LC, organizadores. Como trabalhamos com grupos. Porto Alegre: Artes Médicas; 1997.

24. Leitão GCM. Reflexões sobre gerenciamento. Texto \& contexto enferm. 2001; 10(1):104-15.

25. Passos JP, Ciosak SI. A concepção dos enfermeiros no processo gerencial em Unidade Básica de Saúde. Rev. Esc. Enferm. USP. 2006; 40(4): 464-8.

26. Benito GAV, Becker LC. Atitudes gerenciais do enfermeiro no Programa Saúde da Família: visão da Equipe Saúde da Família. Rev bras enferm. 2007; 60(3):312-6.

Data de recebimento: $17 / 08 / 2010$

Data de aceite: 09/12/2010

Contato com autora responsável: Davi Antonio Brondani Junior.

E-mail: davibrondani@hotmail.com. 